\title{
Foreword
}

\section{The Actions that Define Us: Our Response to Critical Events}

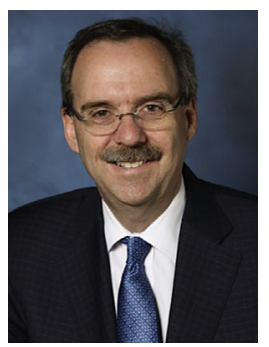

Lee $A$. Fleisher, MD Consulting Editor

As anesthesiologists, we are used to hearing that our job is $99 \%$ boredom and $1 \%$ sheer terror. While many of us believe that this description is far from accurate, our ability to "rescue" a patient who is having a complication and recovery and learn after a critical event is paramount. The method by which we learn to address such events is continuing to evolve as our knowledge of human cognitive traps evolves. In this issue of Anesthesiology Clinics, experts in the field describe some of these general concepts and approaches. This is further enhanced by articles addressing specific events by true experts in their respective fields.

In deciding who could assemble a group of experts and provide a vision for this issue, one name came to mind. Alexander Hannenberg, MD has been a leader in our specialty for decades. I personally met Alex while he was leading the American Society of Anesthesiologists (ASA) efforts in quality and quality measurement for the AMA Physician Consortium for Performance Improvement. He has also served as a past president of the ASA and as its Chief Quality Officer. He is now Clinical Professor of Anesthesiology at Tufts University School of Medicine and is a faculty member in the Safe Surgery Program at Ariadne Labs. He has also given much of his time as a founding board member to the important Lifebox USA. He has assembled 
an amazing group of authors to teach us how to learn and do the best for our patients every day.

Lee A. Fleisher, MD Perelman School of Medicine at University of Pennsylvania 3400 Spruce Street, Dulles 680

Philadelphia, PA 19104, USA

E-mail address:

Lee.Fleisher@uphs.upenn.edu 